\title{
Fast core rotation in red-giant stars as revealed by gravity-dominated mixed modes
}

Paul G. Beck ${ }^{1}$, Josefina Montalban ${ }^{2}$, Thomas Kallinger ${ }^{1,3}$, Joris De Ridder ${ }^{1}$, Conny Aerts ${ }^{1,4}$, Rafael A. García ${ }^{5}$, Saskia Hekker ${ }^{6,7}$, Marc-Antoine Dupret ${ }^{2}$, Benoit Mosser ${ }^{8}$, Patrick Eggenberger ${ }^{9}$, Dennis Stello ${ }^{10}$, Yvonne Elsworth ${ }^{7}$, Søren Frandsen ${ }^{11}$, Fabien Carrier ${ }^{1}$, Michel Hillen ${ }^{1}$, Michael Gruberbauer ${ }^{12}$, Jørgen Christensen-Dalsgaard ${ }^{11}$, Andrea Miglio ${ }^{7}$, Marica Valentini ${ }^{2}$, Timothy R. Bedding ${ }^{10}$, Hans Kjeldsen ${ }^{11}$, Forrest R. Girouard ${ }^{13}$, Jennifer R. Hall ${ }^{13}$ \& Khadeejah A. Ibrahim ${ }^{13}$

When the core hydrogen is exhausted during stellar evolution, the central region of a star contracts and the outer envelope expands and cools, giving rise to a red giant. Convection takes place over much of the star's radius. Conservation of angular momentum requires that the cores of these stars rotate faster than their envelopes; indirect evidence supports this ${ }^{1,2}$. Information about the angular-momentum distribution is inaccessible to direct observations, but it can be extracted from the effect of rotation on oscillation modes that probe the stellar interior. Here we report an increasing rotation rate from the surface of the star to the stellar core in the interiors of red giants, obtained using the rotational frequency splitting of recently detected 'mixed modes' 3,4 . By comparison with theoretical stellar models, we conclude that the core must rotate at least ten times faster than the surface. This observational result confirms the theoretical prediction of a steep gradient in the rotation profile towards the deep stellar interior ${ }^{1,5,6}$.

The asteroseismic approach to studying stellar interiors exploits information from oscillation modes of different radial order $n$ and angular degree $l$, which propagate in cavities extending at different depths ${ }^{7}$. Stellar rotation lifts the degeneracy of non-radial modes, producing a multiplet of $(2 l+1)$ frequency peaks in the power spectrum for each mode. The frequency separation between two mode components of a multiplet is related to the angular velocity and to the properties of the mode in its propagation region. More information on the exploitation of rotational splitting of modes may be found in the Supplementary Information. An important new tool comes from mixed modes that were recently identified in red giants $s^{3,4}$. Stochastically excited solar-like oscillations in evolved $\mathrm{G}$ and $\mathrm{K}$ giant stars ${ }^{8}$ have been well studied in terms of theory ${ }^{9-12}$, and the main results are consistent with recent observations from space-based photometry ${ }^{13,14}$. Whereas pressure modes are completely trapped in the outer acoustic cavity, mixed modes also probe the central regions and carry additional information from the core region, which is probed by gravity modes. Mixed dipole modes $(l=1)$ appear in the Fourier power spectrum as dense clusters of modes around those that are best trapped in the acoustic cavity. These clusters, the components of which contain varying amounts of influence from pressure and gravity modes, are referred to as 'dipole forests'.

We present the Fourier spectra of the brightness variations of stars KIC 8366239 (Fig. 1a), KIC 5356201 (Supplementary Fig. 3a) and KIC 12008916 (Supplementary Fig. 5a), derived from observations with the Kepler spacecraft. The three spectra show split modes, the spherical degree of which we identify as $l=1$. These detected multiplets cannot have been caused by finite mode lifetime effects from mode damping, because that would not lead to a consistent multiplet appearance over several orders such as that shown in Fig. 1. The spacings in period between the multiplet components (Supplementary Fig. 7) are too small to be attributable to consecutive unsplit mixed modes ${ }^{4}$ and do not follow the characteristic frequency pattern of unsplit mixed modes ${ }^{3}$. Finally, the projected surface velocity, $v \sin i$, obtained from ground-based spectroscopy (Table 1), is consistent with the rotational velocity measured from the frequency splitting of the mixed mode that predominantly probes the outer layers. We are thus left with rotation as the only cause of the detected splittings.

The observed rotational splitting is not constant for consecutive dipole modes, even within a given dipole forest (Fig. 1b and Supplementary Figs $3 \mathrm{~b}$ and $5 \mathrm{~b}$ ). The lowest splitting is generally present for the mode at the centre of the dipole forest, which is the mode with the largest amplitude in the outer layers. Splitting increases for modes with a larger gravity component, towards the wings of the dipole mode forest. For KIC 8366239, we find that the average splitting of modes in the wings of the dipole forests is 1.5 times larger than the mean splitting of the centre modes of the dipole forests.

We compared the observations (Fig. 1b) with theoretical predictions for a model representative of KIC 8366239, as defined in the Supplementary Information. The effect of rotation on the oscillation frequencies can be estimated in terms of a weight function, called a rotational kernel $\left(K_{n l}\right)$. From the kernels, it is shown that at least $60 \%$ of the frequency splitting for the $l=1$ mixed modes with a dominant gravity component is produced in the central region of the star (Fig. 2). This substantial core contribution to mixed modes enables us to investigate the rotational properties of the core region, which was hitherto not possible for the Sun, owing to a lack of observed modes that probe the core region (within a radius $r<0.2 R_{\odot}$; ref. 15). The solar rotational profile is known in great detail for only those regions probed by pressure-dominated modes ${ }^{16-18}$. In contrast to these modes in the wings of the dipole forest, only $30 \%$ of the splitting of the centre mode originates from the central region of the star, whereas the outer third by mass of the star contributes $50 \%$ of the frequency splitting. By comparing the rotational velocity derived from the splitting of such pressure-dominated modes with the projected surface velocity from spectroscopy, we find that the asteroseismic value is systematically larger. This offset cannot be explained by inclination of the rotation axis towards the observer alone (Supplementary Tables 1 and 2), but originates from the contribution of the fast-rotating core (Fig. 2). Furthermore, internal non-rigid rotation leads to a larger splitting for modes in the wings of the dipole forest than for centre modes.

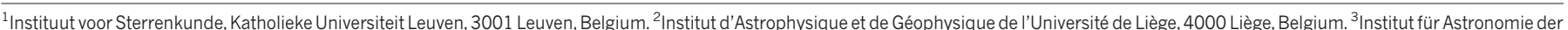
Universität Wien, Türkenschanzstraße 17, 1180 Wien, Austria. ${ }^{4}$ Afdeling Sterrenkunde, Institute for Mathematics Astrophysics and Particle Physics (IMAPP), Radboud University Nijmegen, 6500 GL

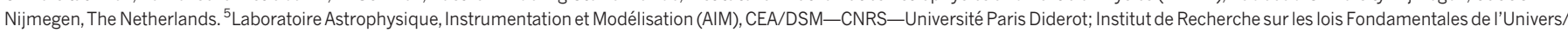

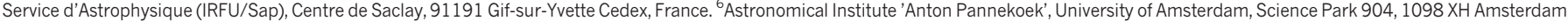

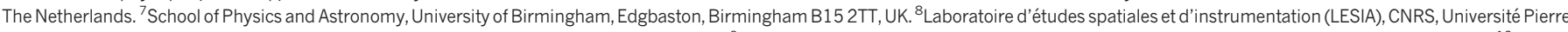

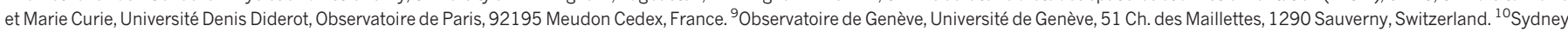
Institute for Astronomy (SIfA), School of Physics, University of Sydney 2006, Australia. ${ }^{11}$ Department of Physics and Astronomy, Aarhus University, DK-8000 Aarhus C, Denmark. ${ }^{12}$ Department of Astronomy and Physics, Saint Marys University, Halifax, NS B3H 3C3, Canada. ${ }^{13}$ Orbital Sciences Corporation/NASA Ames Research Center, Moffett Field, 94035 California, USA. 


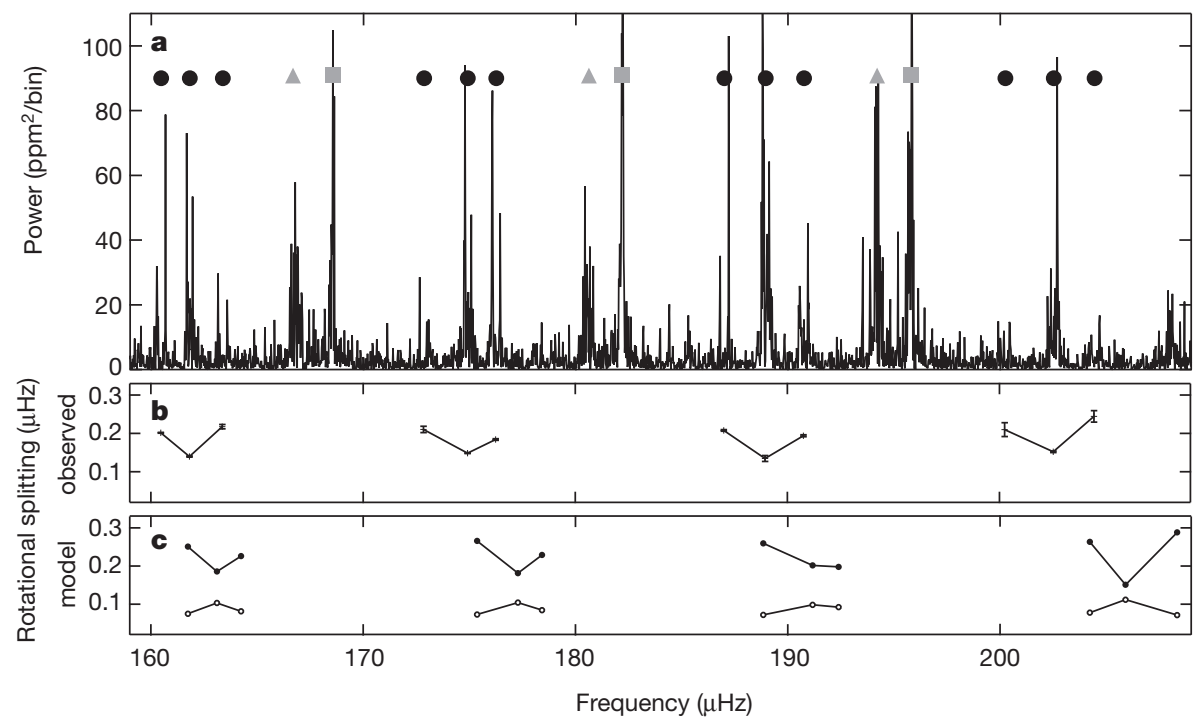

Figure $1 \mid$ Oscillation spectrum of KIC 8366239. a, Grey squares, radial modes; grey triangles, $l=2$ modes; black circles, $l=1$ rotational multiplets. A zoom on the region of $185-195 \mu \mathrm{Hz}$ and the analysis of the comb-like structure of the oscillation spectrum in an échelle diagram are shown in the online material (Supplementary Figs 1 and 2). The spectral window of the Fourier analysis can be found in the online material (Supplementary Fig. 11). The $y$-axis indicates the flux variation power in parts-per-million squared for each frequency bin. $\mathbf{b}$, The observed rotational splitting for individual $l=1$ modes. Error bars, standard deviation of the measured rotational splitting of dipole modes. Similar analyses of the stars KIC 5356201 (Supplementary Figs 3 and 4) and KIC 12008916 (Supplementary Figs 5 and 6) are discussed in the online material. c, Theoretically predicted rotational splitting assuming two different

For a model rotating rigidly, the reverse behaviour is expected (Fig. 1c). Because the prediction for the rigidly rotating model is incompatible with the observed trends of the splittings (Fig. 1b) but can be well reproduced qualitatively under the assumption of non-rigid rotation (Fig. 1c), we conclude that the three stars investigated here (see Table 1, last column) rotate non-rigidly, with the central region rotating much faster than the surface.

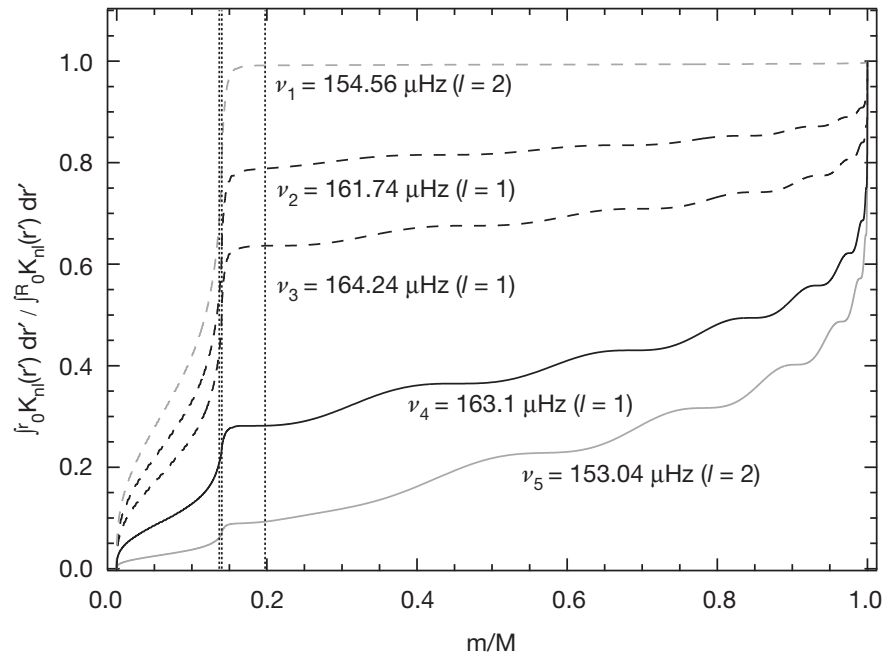

Figure 2 Contributions to the total rotational splitting. Partial integrals of normalized rotation kernels, illustrating the contribution from different regions to the rotational splitting for pressure-dominated modes $\left(v_{4}, v_{5}\right.$; solid lines) and gravity-dominated modes $\left(v_{1}, v_{2}, v_{3}\right.$; dashed lines) of degree $l=1$ and $l=2$, as a function of the stellar mass-fraction, $\mathrm{m} / \mathrm{M}$. The kernels have been calculated for modes from a representative model of KIC 8366239, as defined in the Supplementary Information, with oscillation frequencies given. Vertical dotted lines, left to right: boundary of the helium core, the hydrogen-burning shell and the bottom of the convective envelope. rotation laws. The values are calculated for a representative model of KIC 8366239 as defined in the Supplementary Information. Solid circles, splitting for non-rigid rotation for the case of a core rotation ten times faster than the surface rotation of $2.5 \mathrm{~km} \mathrm{~s}^{-1}$ resembles the observations qualitatively well. Open circles, theoretical splittings for rigid rotation and an equatorial surface rotation velocity of $3 \mathrm{~km} \mathrm{~s}^{-1}$ show a trend opposite to the observed one, with the largest splitting in the centre of the dipole forest and lower splitting in gravity-dominated modes. In the case of rigid rotation, the variable splitting is governed purely by the variation of the Ledoux ${ }^{24}$ constant across the dipole forest (Supplementary Fig. 8). Because the representative model (c) has not been corrected for surface effects, there is a slight offset to the observations (b).

The above interpretation is consistent with the correlation between the mode lifetime and the corresponding rotational splitting that has been observed in our data. Mixed modes in the wings of the dipole forest are predicted to have large amplitudes in the central regions of the star and, therefore, larger values of inertia and lifetime. These modes have narrower mode profiles in the frequency spectrum than have the centre modes that are predominantly trapped in the outer cavity $^{11}$. This behaviour of the mode profiles (Supplementary Fig. 1)

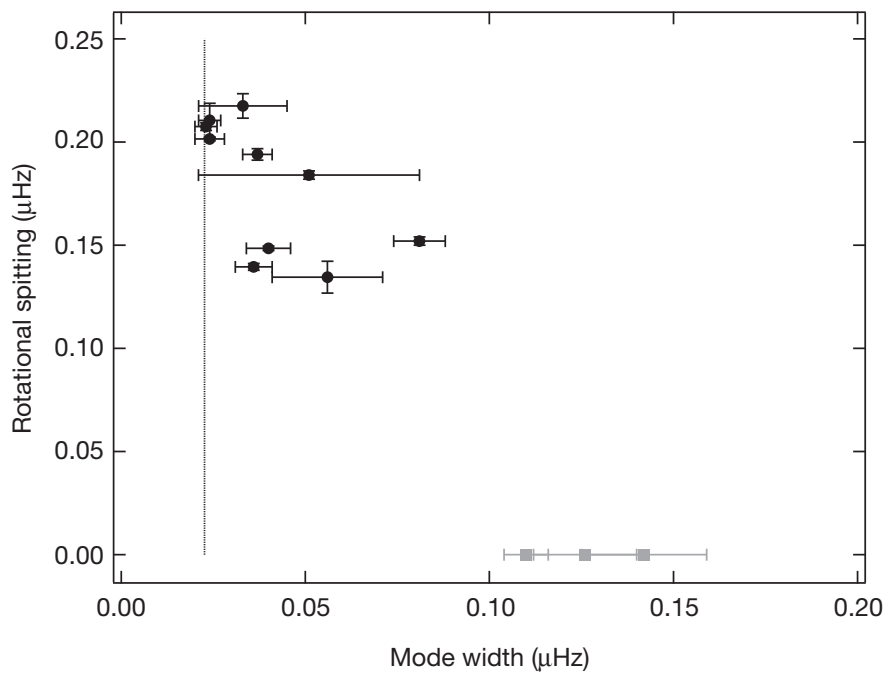

Figure $3 \mid$ Rotational splitting versus mode linewidth for KIC 8366239. The lifetime is inversely proportional to the mode linewidth. Black circles, $l=1$ modes. Grey squares, linewidth of the pure acoustic radial modes $(l=0)$, for comparison. Dotted vertical line, formal frequency resolution. Error bars, standard deviation of the measured rotational splitting and mode width of dipole modes. Similar diagrams for the two other stars from Table 1 are shown in Supplementary Figs 9 and 10, respectively. 
Table 1 | Observational parameters of three stars, showing rotational splitting.

\begin{tabular}{|c|c|c|c|c|c|c|c|c|}
\hline KIC & $v_{\max }(\mu \mathrm{Hz})$ & $R\left(R_{\odot}\right)$ & $\Delta \boldsymbol{P}_{\text {obs }}(\mathrm{s})$ & $T_{\text {eff }}(\mathrm{K})$ & $\begin{array}{c}v \sin i \\
\left(\mathrm{~km} \mathrm{~s}^{-1}\right)\end{array}$ & $\begin{array}{l}\text { Asteros. rot. } \\
\text { vel. }\left(\mathrm{km} \mathrm{s}^{-1}\right)\end{array}$ & $\begin{array}{c}I=1 \text { min rot. } \\
\text { split. }(\mu \mathrm{Hz})\end{array}$ & $\begin{array}{l}\text { Averaged split. ratio } \\
\text { for dipole modes }\end{array}$ \\
\hline 8366239 & $182 \pm 1$ & $5.30 \pm 0.08$ & $56 \pm 11$ & $4980 \pm 120$ & $<1$ & 3.9 & $0.135 \pm 0.008$ & 1.5 \\
\hline 5356201 & $209.7 \pm 0.7$ & $4.47 \pm 0.03$ & $50 \pm 10$ & $4840 \pm 90$ & 2.4 & 3.8 & $0.154 \pm 0.003$ & 1.7 \\
\hline 12008916 & $159.9 \pm 0.6$ & $5.18 \pm 0.05$ & $52 \pm 7$ & $4830 \pm 100$ & - & 5.7 & $0.200 \pm 0.001$ & 1.8 \\
\hline
\end{tabular}

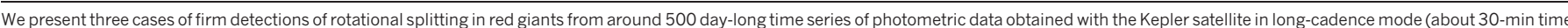

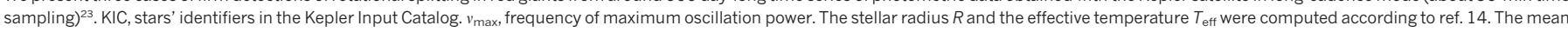

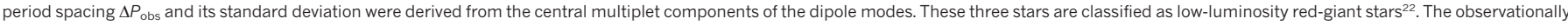

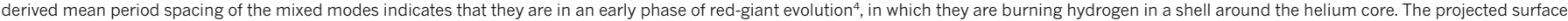

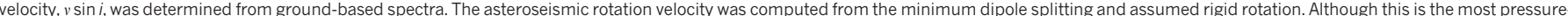

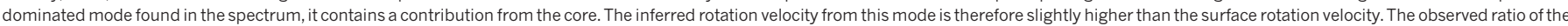

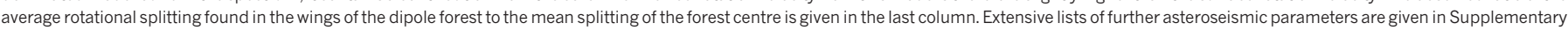
Table 1 and 2.

was recently confirmed by observations ${ }^{3,4}$. We observe that short-lived modes (with broader profiles) exhibit smaller rotational splitting in KIC 8366239 (Fig. 3) and the two other stars from Table 1 (Supplementary Figs 9 and 10). With increasing lifetime (dominant gravity component) we see a substantial increase in the size of the rotational splitting of the modes. The frequencies of the narrowest modes are mainly affected by the rotation in the central regions of the star. Taking a representative model for KIC 8366239, and assuming that the convective envelope rotates rigidly and that the radiative interior rotates rigidly but faster than the convective envelope, we find that the observed splitting ratio of 1.5 (Table 1 ) is obtained from a core rotating at least ten times faster than the surface.

So far, our understanding of the evolution of the angular momentum as a function of the evolutionary stage is still poor. Before this study, it was possible to deliver evidence of non-rigid rotation in only two corehydrogen-burning massive stars, for which the seismic analysis revealed a near-core rotation rate about three to five times faster than the envelope rotation rate ${ }^{19,20}$. Claims of non-rigid rotation in white dwarfs have also been discussed ${ }^{21}$. The low-luminosity red giants ${ }^{22}$ discussed here are in the early phase of hydrogen-shell burning, and have a core-to-envelope rotation rate more than twice as high as those of the massive stars ${ }^{19,20}$. When these stars enter the subsequent phase of core-helium burning, we believe that the core will undergo a slight expansion and the envelope will shrink, leading to a surface rotation on the horizontal branch larger than that on the red-giant branch ${ }^{1}$. The basic assumption of conservation of angular momentum predicts a rotational gradient between surface and core that is less steep than the one detected. Exploiting rotational splitting of mixed modes in a large sample of red giants in various evolutionary stages will provide an excellent tool to inspect how the internal angular-momentum distribution evolves with time towards the end of the life of the star.

Received 29 July; accepted 29 September 2011.

Published online 7 December 2011.

1. Sills, A. \& Pinsonneault, M. H. Rotation of horizontal-branch stars in globular clusters. Astrophys. J. 540, 489-503 (2000).

2. Sweigart, A. V. \& Mengel, J. G. Meridional circulation and $\mathrm{CNO}$ anomalies in red giant stars. Astrophys. J. 229, 624-641 (1979).

3. Beck, P. G. et al. Kepler detected gravity-mode period spacings in a red giant star. Science 332, 205 (2011).

4. Bedding, T. R. et al. Gravity modes as a way to distinguish between hydrogen- and helium-burning red giant stars. Nature 471, 608-611 (2011).

5. Zahn, J.-P. Circulation and turbulence in rotating stars. Astron. Astrophys. 265, 115-132 (1992).

6. Eggenberger, P. et al. Effects of rotation on the evolution and asteroseismic properties of red giants. Astron. Astrophys. 509, A72 (2010).

7. Aerts, C., Christensen-Dalsgaard, J. \& Kurtz, D. W. Asteroseismology Ch. 3 (SpringerVerlag, 2010).

8. De Ridder, J. et al. Non-radial oscillation modes with long lifetimes in giant stars. Nature 459, 398-400 (2009).
9. Dziembowski, W. A. et al. Oscillations of $\alpha \mathrm{UMa}$ and other red giants. Mon. Not. R. Astron. Soc. 328, 601-610 (2001).

10. Christensen-Dalsgaard, J. Physics of solar-like oscillations. Sol. Phys. 220, 137-168 (2004).

11. Dupret, M.-A. et al. Theoretical amplitudes and lifetimes of non-radial solar-like oscillations in red giants. Astron. Astrophys. 506, 57-67 (2009).

12. Montalbán, J., Miglio, A., Noels, A., Scuflaire, R. \& Ventura, P. Seismic diagnostics of red giants: first comparison with stellar models. Astrophys. J. 721, L182-L188 (2010).

13. Mosser, B. et al. The universal red-giant oscillation pattern. An automated determination with CoRoT data. Astron. Astrophys. 525, L9 (2011).

14. Kallinger, T. et al. Asteroseismology of red giants from the first four months of Kepler data: fundamental stellar parameters. Astron. Astrophys. 522, A1 (2010).

15. García, R. A. et al. Tracking solar gravity modes: the dynamics of the solar core. Science 316, 1591-1593 (2007).

16. Elsworth, Y. et al. Slow rotation of the Sun's interior. Nature 376, 669-672 (1995).

17. Chaplin, W. J. et al. Rotation of the solar core from BiSON and LOWL frequency observations. Mon. Not. R. Astron. Soc. 308, 405-414 (1999).

18. Thompson, M. J. et al. The internal rotation of the Sun. Annu. Rev. Astron. Astrophys. 41, 599-643 (2003).

19. Aerts, C. etal. Asteroseismology of HD 129929: core overshooting and nonrigid rotation. Science 300, 1926-1928 (2003).

20. Pamyatnykh, A. A. et al. Asteroseismology of the $\beta$ Cephei star $v$ Eridani: interpretation and applications of the oscillation spectrum. Mon. Not. R. Astron. Soc. 350, 1022-1028 (2004).

21. Kawaler, S. D., Sekii, T. \& Gough, D. Prospects for measuring differential rotation in white dwarfs through asteroseismology. Astrophys. J. 516, 349-365 (1999).

22. Bedding, T. R. et al. Solar-like oscillations in low-luminosity red giants: first results from Kepler. Astrophys. J. Lett. 713, L176-L181 (2010).

23. García, R. A. et al. Preparation of Kepler light curves for asteroseismic analyses. Mon. Not. R. Astron. Soc. 414, L6-L10 (2011).

24. Ledoux, P. The nonradial oscillations of gaseous stars and the problem of beta Canis Majoris. Astrophys. J. 114, 373-384 (1951).

Supplementary Information is linked to the online version of the paper at www.nature.com/nature.

Acknowledgements We acknowledge the work of the team behind Kepler. Funding for the Kepler Mission is provided by NASA's Science Mission Directorate. P.G.B. and C.A. were supported by the European Community's Seventh Framework Programme (ERC grantPROSPERITY); J.D.R. and T.K. were supported by the Fund for Scientific Research, Flanders. S.H. was supported by the Netherlands Organisation for Scientific Research. J.M. and M.V. were supported by the Belgian Science Policy Office. The work is partly based on observations with the High Efficiency and Resolution Mercator Echelle Spectrograph at the Mercator Telescope, which is operated at La Palma in Spain by the Flemish Community.

Author Contributions P.G.B., T.K., J.D.R., C.A., R.A.G., S.H., B.M., Y.E., S.F., F.C. and M.G. measured the mode parameters, and derived and interpreted the rotational splitting and period spacings. J.M., M.-A.D., P.E., J.C.-D. and A.M. calculated stellar models and provided theoretical interpretation of the rotational splitting. M.H. and M.V. observed and analysed the spectra. J.D.R., S.H., S.F., Y.E., D.S., T.R.B., H.K., F.R.G., J.R.H. and K.A.I. contributed to the coordination of the project, including the acquisition and distribution of the data. C.A. defined and supervised the research. All authors discussed the results and commented on the manuscript.

Author Information Reprints and permissions information is available at www.nature.com/reprints. The authors declare no competing financial interests. Readers are welcome to comment on the online version of this article at www.nature.com/nature. Correspondence and requests for materials should be addressed to P.G.B. (paul.beck@ster.kuleuven.be). 